\title{
Non-invariant submanifolds of locally decomposable golden Riemannian manifolds
}

Received: 12 July 2020 / Accepted: 8 December 2020 / Published online: 12 January 2021

(C) The Author(s) 2021

\begin{abstract}
In this paper, we investigate any non-invariant submanifold of a locally decomposable golden Riemannian manifold in the case that the rank of the set of tangent vector fields of the induced structure on the submanifold by the golden structure of the ambient manifold is less than or equal to the codimension of the submanifold.
\end{abstract}

Mathematics Subject Classification $53 \mathrm{C} 15 \cdot 53 \mathrm{C} 25 \cdot 53 \mathrm{C} 40$

\section{Introduction}

Recently, the notion of a golden structure has widely appeared in the literature of special geometric structures on manifolds and it has been studied by many geometers. At first, golden structures on manifolds were examined with the help of the related almost product structures by C. E. Hreţcanu and M. C. Crâş̧ăreanu in [5] including basic properties; the integrability and parallelism conditions; some examples; the treatment of connections in principal and tangent bundles in terms of golden structures. In addition, by endowing a golden structure with a main geometric object, namely a Riemannian metric, the concepts of a golden Riemannian structure and a golden Riemannian manifold were introduced and their some fundamental properties were established. In this sense, similar studies were considered for silver structures [19] and bronze structures [20] on manifolds. From the different viewpoint, A. Gezer, N. Cengiz, and A. Salimov investigated the integrability of golden Riemannian structures, some properties of twin golden Riemannian metrics, and the Riemannian curvature tensor of locally decomposable golden Riemannian manifolds by using a particular operator in [7]. In [23], B. Şahin and M. A. Akyol defined the notion of a golden map between two golden Riemannian manifolds and showed that it is harmonic. In addition, the authors discussed the constancy of some maps from golden Riemannian manifolds to $C^{\infty}$-differentiable manifolds endowed with certain special geometric structures. Golden structures have been also explored in various contexts [3,8,9,18].

The first research on the differential geometry of submanifolds in golden Riemannian manifolds was initiated by C. E. Hreţcanu and M. C. Crâşmăreanu in [14,15]. Herein to begin with, several properties of the induced structure on any submanifold in a golden Riemannian manifold were established. Second, it was proven that any invariant submanifold of a golden Riemannian manifold is also a golden Riemannian manifold. Finally, it was shown that the Nijenhuis tensor of the induced structure vanishes identically on

M. Gök (凶)

Department of Design, Sivas Cumhuriyet University, 58140 Sivas, Turkey

E-mail: mustafa.gok@email.com

E. Kılıç

Department of Mathematics, İnönü University, 44280 Malatya, Turkey

E-mail: erol.kilic@inonu.edu.tr 
invariant submanifolds in the event that the ambient manifold is a locally decomposable golden Riemannian manifold. Since then, it has made great progress. The different kinds of classes of submanifolds in golden Riemannian manifolds were defined according to the behavior of their tangent bundles with respect to the action of the golden structure of the ambient manifold and searched by several geometers. The concept of a semi-invariant submanifold in golden Riemannian manifolds was given by F. E. Erdoğan and C. Yıldırım [6] as a generalization of both invariant and anti-invariant submanifolds, and then, the geometry of the distributions involved in its definition was studied. M. Gök, S. Keleş, and E. Kılıç examined some characterizations of semi-invariant submanifolds of a golden Riemannian manifold and their associated distributions in [10]. The authors also investigated invariant submanifolds of golden Riemannian manifolds in [11]. The notions of a slant submanifold, a semi-slant submanifold, and a hemi-slant submanifold in golden Riemannian manifolds were introduced by C. E. Hreţcanu and A. M. Blaga in [4,12,13]. In the three above mentioned papers, some characterizations were first found for any submanifold in a golden Riemannian manifold to be slant, semi-slant, or hemi-slant. Next, the integrability conditions of the distributions in their definitions were obtained. After that, some necessary conditions were got for such classes of submanifolds to be mixed totally geodesic. Lastly, the parallelism of canonical structures induced on these submanifolds by the golden structure of the ambient manifold was analyzed.

On the other hand, inspired by [5] being the fundamental paper regarding golden structures on manifolds and S. Kalia's own definition of the bronze mean in given [17] different from that of [16], B. Şahin [22] introduced the concept of an almost poly-Norden semi-Riemannian manifold, and then, S. Y. Perktaş [21] investigated two well-known classes of submanifolds in almost poly-Norden Riemannian manifolds, namely invariant and anti-invariant submanifolds, and obtained important results on them.

The main purpose of this paper is to search non-invariant submanifolds of a locally decomposable golden Riemannian manifold with the aid of induced structures on them by the golden structure of the ambient manifold.

The paper has three sections and is organized as follows: Sect. 2 is devoted to preliminaries containing basic definitions, concepts, formulas, notations, and results for golden Riemannian manifolds and their submanifolds. Section 3 is concerned with an examination of non-invariant submanifolds of a locally decomposable golden Riemannian manifold. We give some necessary conditions for any non-invariant submanifold in a locally decomposable golden Riemannian manifold to be totally geodesic or minimal. Besides, we get some results on the non-invariant submanifold in the event that the tangent vector fields of the induced structure are linearly dependent.

\section{Preliminaries}

This section deals with some known fundamental definitions, concepts, formulas, notations, and results on golden Riemannian manifolds and their submanifolds.

A non-trivial tensor field $\bar{\Phi}$ of type $(1,1)$ on a $C^{\infty}$-differentiable real manifold $\bar{M}$ is called a golden structure if it satisfies the equation:

$$
\bar{\Phi}^{2}=\bar{\Phi}+I
$$

where $I$ is the identity operator on the Lie algebra $\Gamma(T \bar{M})$ of differentiable vector fields on $\bar{M}$. Also, we say that $\bar{M}$ is a golden manifold. If $(\bar{M}, \bar{g})$ is a Riemannian manifold endowed with a golden structure $\bar{\Phi}$, such that $\bar{g}$ and $\bar{\Phi}$ verify the relation:

$$
\bar{g}(\bar{\Phi} X, Y)=\bar{g}(X, \bar{\Phi} Y)
$$

for any vector fields $X, Y \in \Gamma(T \bar{M})$, and then, the pair $(\bar{g}, \bar{\Phi})$ and the triple $(\bar{M}, \bar{g}, \bar{\Phi})$ are named a golden Riemannian structure and a golden Riemannian manifold, respectively. The eigenvalues of the golden structure $\bar{\Phi}$ are the roots of the quadratic equation $x^{2}-x-1=0$; that is, $\phi=\frac{1+\sqrt{5}}{2}$ and $1-\phi=\frac{1-\sqrt{5}}{2}$, where the former is the golden ratio. The inverse $\bar{\Phi}^{-1}$ of the golden structure $\bar{\Phi}$ is given by $\bar{\Phi}^{-1}=\bar{\Phi}-I$ and satisfies the equation $\left(\bar{\Phi}^{-1}\right)^{2}=-\bar{\Phi}^{-1}+I$, so it is not a golden structure $[5,14,15]$. 
Let $M$ be an $n$-dimensional submanifold of codimension $r$, isometrically immersed in any $m$-dimensional golden Riemannian manifold $(\bar{M}, \bar{g}, \bar{\Phi})$. We denote by $T_{p} M$ and $T_{p} M^{\perp}$ its tangent and normal spaces at a point $p \in M$, respectively. Then, the tangent space $T_{p} \bar{M}$ has the decomposition:

$$
T_{p} \bar{M}=T_{p} M \oplus T_{p} M^{\perp}
$$

for each point $p \in M$. The induced Riemannian metric $g$ on $M$ is given by:

$$
g(X, Y)=\bar{g}\left(i_{*} X, i_{*} Y\right)
$$

for any vector fields $X, Y \in \Gamma(T M)$, where $i_{*}$ is the differential of the immersion $i: M \longrightarrow \bar{M}$. Now, let us consider a local orthonormal frame $\left\{N_{1}, \ldots, N_{r}\right\}$ of the normal bundle $T M^{\perp}$. For every vector field $X \in \Gamma(T M)$, the vector fields $\bar{\Phi}\left(i_{*} X\right)$ and $\bar{\Phi}\left(N_{\alpha}\right)$ on the ambient manifold $\bar{M}$ can be expressed in the following forms:

$$
\bar{\Phi}\left(i_{*} X\right)=i_{*}(\Phi(X))+\sum_{\alpha=1}^{r} u_{\alpha}(X) N_{\alpha}
$$

and

$$
\bar{\Phi}\left(N_{\alpha}\right)=\varepsilon i_{*}\left(\xi_{\alpha}\right)+\sum_{\beta=1}^{r} a_{\alpha \beta} N_{\beta}, \varepsilon= \pm 1
$$

respectively, where $\Phi$ is a tensor field of type $(1,1)$ on $M, \xi_{\alpha}$ 's are tangent vector fields on $M, u_{\alpha}$ 's are differential 1-forms on $M$, and $\left(a_{\alpha \beta}\right)$ is a matrix of type $r \times r$ of real functions on $M$ for any $\alpha, \beta \in\{1, \ldots, r\}$. Hence, we get a structure $\left(\Phi, g, u_{\alpha}, \varepsilon \xi_{\alpha},\left(a_{\alpha \beta}\right)_{r \times r}\right)$ induced on $M$ by the golden Riemannian structure $(\bar{g}, \bar{\Phi})$. We denote by $\bar{\nabla}$ and $\nabla$ the Levi-Civita connections on the ambient manifold $\bar{M}$ and the submanifold $M$, respectively. Then, the Gauss and Weingarten formulas of $M$ in $\bar{M}$ are given, respectively, by:

$$
\bar{\nabla}_{i_{*} X} i_{*} Y=i_{*} \nabla_{X} Y+\sum_{\alpha=1}^{r} h_{\alpha}(X, Y) N_{\alpha}
$$

and

$$
\bar{\nabla}_{i_{*} X} N_{\alpha}=-i_{*} A_{\alpha} X+\sum_{\beta=1}^{r} l_{\alpha \beta}(X) N_{\beta}
$$

for any vector fields $X, Y \in \Gamma(T M)$, where $h_{\alpha}$ 's are the second fundamental tensors corresponding to $N_{\alpha}$ 's, $A_{\alpha}$ 's are the shape operators in the direction of $N_{\alpha}$ 's, and $l_{\alpha \beta}$ 's are the differential 1-forms on $M$ corresponding to the normal connection $\nabla^{\perp}$. Also, the following relations hold:

$$
\begin{aligned}
h(X, Y) & =\sum_{\alpha=1}^{r} h_{\alpha}(X, Y) N_{\alpha}, \\
h_{\alpha}(X, Y) & =h_{\alpha}(Y, X), \\
h_{\alpha}(X, Y) & =g\left(A_{\alpha} X, Y\right), \\
\nabla_{X}^{\perp} N_{\alpha} & =\sum_{\beta=1}^{r} l_{\alpha \beta}(X) N_{\beta}
\end{aligned}
$$

and

$$
l_{\alpha \beta}=-l_{\beta \alpha}
$$

for any vector fields $X, Y \in \Gamma(T M)$ and $\alpha, \beta \in\{1, \ldots, r\}$ [14]. 
As it is well known, the submanifold $M$ is called totally geodesic if $h=0$. Besides, the mean curvature vector $H$ of $M$ is defined by:

$$
H=\frac{1}{n} \sum_{i=1}^{n} h\left(E_{i}, E_{i}\right)
$$

where $\left\{E_{1}, \ldots, E_{n}\right\}$ is a local orthonormal frame of the tangent bundle $T M$. If $H=0$, then $M$ is said to be a minimal submanifold. The submanifold $M$ is named totally umbilical if $h(X, Y)=g(X, Y) H$ for any vector fields $X, Y \in \Gamma(T M)$ [24].

The triple $(\bar{M}, \bar{g}, \bar{\Phi})$ is called a locally decomposable golden Riemannian manifold if the golden structure $\bar{\Phi}$ is parallel with respect to the Levi-Civita connection $\bar{\nabla}$, i.e., the covariant derivative $\bar{\nabla} \bar{\Phi}$ is identically zero [13].

\section{Submanifolds of golden Riemannian manifolds}

In this section, we focus on a study of non-invariant submanifolds in locally decomposable golden Riemannian manifolds. We give some necessary conditions for any non-invariant submanifold of a locally decomposable golden Riemannian manifold to be totally geodesic or totally umbilical. Besides, we investigate the case when the rank of the matrix $\left(\xi_{1} \cdots \xi_{r}\right)$ is less than its codimension $r$. Herein, we first obtain some necessary conditions for each of the second fundamental tensors $h_{\alpha}$ 's to be zero, where $1 \leq \alpha \leq \operatorname{rank}\left(\xi_{1} \cdots \xi_{r}\right)$. Later, we get a result on each of the second fundamental tensors $h_{\alpha}$ 's.

Lemma 3.1 [14] Let $M$ be an n-dimensional submanifold of codimension $r$, isometrically immersed in any golden Riemannian manifold $(\bar{M}, \bar{g}, \bar{\Phi})$. Then, induced structure $\left(\Phi, g, u_{\alpha}, \varepsilon \xi_{\alpha},\left(a_{\alpha \beta}\right)_{r \times r}\right)$ on $M$ by the golden Riemannian structure $(\bar{g}, \bar{\Phi})$ satisfies the following relations:

$$
\begin{aligned}
\Phi^{2}(X) & =\Phi(X)+X-\varepsilon \sum_{\alpha=1}^{r} u_{\alpha}(X) \xi_{\alpha}, \\
u_{\alpha}(\Phi(X)) & =\left(1-a_{\alpha \alpha}\right) u_{\alpha}(X), \\
a_{\alpha \beta} & =a_{\beta \alpha}, \\
u_{\beta}\left(\xi_{\alpha}\right) & =\varepsilon\left(\delta_{\alpha \beta}+a_{\alpha \beta}-\sum_{\gamma=1}^{r} a_{\alpha \gamma} a_{\beta \gamma}\right), \\
\Phi\left(\xi_{\alpha}\right) & =\xi_{\alpha}-\sum_{\beta=1}^{r} a_{\alpha \beta} \xi_{\beta}, \\
u_{\alpha}(X) & =\varepsilon g\left(X, \xi_{\alpha}\right), \\
g(\Phi(X), Y) & =g(X, \Phi(Y))
\end{aligned}
$$

and

$$
g(\Phi(X), \Phi(Y))=g(\Phi(X), Y)+g(X, Y)-\sum_{\alpha=1}^{r} u_{\alpha}(X) u_{\alpha}(Y)
$$

for any vector fields $X, Y \in \Gamma(T M)$.

Proposition 3.2 [14] Let $M$ be an n-dimensional submanifold of codimension $r$, isometrically immersed in any golden Riemannian manifold $(\bar{M}, \bar{g}, \bar{\Phi})$. If the tangent vector fields $\xi_{1}, \ldots, \xi_{r}$ are linearly independent, then the differential 1 -forms $u_{1}, \ldots, u_{r}$ are also linearly independent. 
Lemma 3.3 [14] Let $M$ be an n-dimensional submanifold of codimension $r$, isometrically immersed in any locally decomposable golden Riemannian manifold $(\bar{M}, \bar{g}, \bar{\Phi})$. Then, we have the following relations:

$$
\begin{aligned}
\left(\nabla_{X} \Phi\right) Y & =\varepsilon \sum_{\alpha=1}^{r} h_{\alpha}(X, Y) \xi_{\alpha}+\sum_{\alpha=1}^{r} u_{\alpha}(Y) A_{\alpha} X \\
\left(\nabla_{X} u_{\alpha}\right) Y & =-h_{\alpha}(X, \Phi Y)+\sum_{\beta=1}^{r} u_{\beta}(Y) l_{\alpha \beta}(X)+\sum_{\beta=1}^{r} h_{\beta}(X, Y) a_{\alpha \beta}, \\
\nabla_{X} \xi_{\alpha} & =-\varepsilon \Phi\left(A_{\alpha} X\right)+\varepsilon \sum_{\beta=1}^{r} a_{\alpha \beta} A_{\beta} X+\sum_{\beta=1}^{r} l_{\alpha \beta}(X) \xi_{\beta}
\end{aligned}
$$

and

$$
X\left(a_{\alpha \beta}\right)=-\varepsilon h_{\beta}\left(X, \xi_{\alpha}\right)-\varepsilon h_{\alpha}\left(X, \xi_{\beta}\right)-\sum_{\gamma=1}^{r} a_{\alpha \gamma} l_{\gamma \beta}(X)-\sum_{\gamma=1}^{r} a_{\beta \gamma} l_{\gamma \alpha}(X)
$$

for any vector fields $X, Y \in \Gamma(T M)$.

Theorem 3.4 Let $M$ be an n-dimensional submanifold of codimension $r$, isometrically immersed in any locally decomposable golden Riemannian manifold $(\bar{M}, \bar{g}, \bar{\Phi})$. If the tangent vector fields $\xi_{1}, \ldots, \xi_{r}$ are linearly independent and $\nabla \Phi=0$, then $M$ is a totally geodesic submanifold.

Proof Taking account of the assumption that $\nabla \Phi=0$, we obtain from (11), (20), and (23) that:

$$
\sum_{\alpha=1}^{r} u_{\alpha}(Y) h_{\alpha}(X, Z)=-\sum_{\alpha=1}^{r} u_{\alpha}(Z) h_{\alpha}(X, Y)
$$

for any vector fields $X, Y, Z \in \Gamma(T M)$. Using (10) in (27), we get:

$$
\sum_{\alpha=1}^{r} u_{\alpha}(Y) h_{\alpha}(X, Z)=\sum_{\alpha=1}^{r} u_{\alpha}(Z) h_{\alpha}(X, Y)
$$

for any vector fields $X, Y, Z \in \Gamma(T M)$. Hence, it follows from (27) and (28) that:

$$
\sum_{\alpha=1}^{r} u_{\alpha}(Y) h_{\alpha}(X, Z)=0
$$

On the other hand, Proposition 3.2 states that if $\xi_{1}, \ldots, \xi_{r}$ are linearly independent tangent vector fields, then the differential 1-forms $u_{1}, \ldots, u_{r}$ are linearly independent. Thus, we infer from (29) that $h_{\alpha}(X, Z)=0$ for any $\alpha \in\{1, \ldots, r\}$. Taking account of (9), we have $h=0$. In other words, $M$ is a totally geodesic submanifold.

Lemma 3.5 [15] Let $M$ be an $n$-dimensional submanifold of codimension $r$, isometrically immersed in any golden Riemannian manifold $(\bar{M}, \bar{g}, \bar{\Phi})$. If the tangent vector fields $\xi_{1}, \ldots, \xi_{r}$ are linearly independent, then the trace of the induced structure $\Phi$ is given as follows:

$$
\operatorname{tr}(\Phi)=\left\{\begin{array}{l}
r-\operatorname{tr}\left(a_{\alpha \beta}\right)+\sum_{A=r+1}^{n} \lambda_{A}, r<n \\
r-\operatorname{tr}\left(a_{\alpha \beta}\right), r=n
\end{array}\right.
$$

where $\lambda_{A} \in\{\phi, 1-\phi\}$.

Lemma 3.6 [2] Let $M$ be an n-dimensional submanifold of codimension $r$, isometrically immersed in any golden Riemannian manifold $(\bar{M}, \bar{g}, \bar{\Phi})$. If the tangent vector fields $\xi_{1}, \ldots, \xi_{r}$ are linearly independent and $\nabla \Phi=0$, then $\operatorname{tr}\left(a_{\alpha \beta}\right)$ is constant. 
Theorem 3.7 [2] Let $M$ be an n-dimensional submanifold of codimension $r$, isometrically immersed in any locally decomposable golden Riemannian manifold $(\bar{M}, \bar{g}, \bar{\Phi})$. If the tangent vector fields $\xi_{1}, \ldots, \xi_{r}$ are linearly independent, $\operatorname{tr}(\Phi)$ is constant, and $M$ is totally umbilical, then $M$ is totally geodesic.

Theorem 3.8 [2] Let $M$ be an n-dimensional submanifold of codimension $r$, isometrically immersed in any locally decomposable golden Riemannian manifold $(\bar{M}, \bar{g}, \bar{\Phi})$. If the tangent vector fields $\xi_{1}, \ldots, \xi_{r}$ are linearly independent, $\operatorname{tr}\left(a_{\alpha \beta}\right)$ is constant, and $\sum_{i=1}^{n}\left(\nabla_{e_{i}} \Phi\right) e_{i}=0$, then $M$ is minimal, where $\left\{e_{1}, \ldots, e_{n}\right\}$ is an orthonormal basis of the tangent space $T_{p} M$ at a point $p \in M$.

Lemma 3.9 Let $M$ be an $n$-dimensional submanifold of codimension $r$, isometrically immersed in any golden Riemannian manifold $(\bar{M}, \bar{g}, \bar{\Phi})$. If $a_{\alpha \beta}=\lambda_{\alpha} \delta_{\alpha \beta}, \lambda_{\alpha} \in(1-\phi, \phi)$ for any $\alpha, \beta \in\{1, \ldots, r\}$, then the following assertions are valid:

(a) The tangent vector fields $\xi_{1}, \ldots, \xi_{r}$ are linearly independent,

(b) The tangent vector field $\xi_{\alpha}$ is an eigenvector corresponding to the eigenvalue $1-\lambda_{\alpha}$ for any $\alpha \in\{1, \ldots, r\}$.

Proof We assume that $a_{\alpha \beta}=\lambda_{\alpha} \delta_{\alpha \beta}, \lambda_{\alpha} \in(1-\phi, \phi)$ for any $\alpha, \beta \in\{1, \ldots, r\}$. By a straightforward calculation, we infer from (18) that:

$$
u_{\beta}\left(\xi_{\alpha}\right)=\varepsilon \delta_{\alpha \beta}\left(1+\lambda_{\alpha}-\lambda_{\alpha} \lambda_{\beta}\right) .
$$

On the other hand, it can be easily seen from (20) that $g\left(\xi_{\alpha}, \xi_{\beta}\right)=\varepsilon u_{\beta}\left(\xi_{\alpha}\right)$. Hence, we obtain:

$$
g\left(\xi_{\alpha}, \xi_{\beta}\right)=\delta_{\alpha \beta}\left(1+\lambda_{\alpha}-\lambda_{\alpha} \lambda_{\beta}\right) .
$$

In addition, taking account of (17), (31) takes the form:

$$
g\left(\xi_{\alpha}, \xi_{\beta}\right)=\delta_{\alpha \beta}\left(1+\lambda_{\alpha}-\lambda_{\alpha}^{2}\right)
$$

If we write $\sum_{\beta=1}^{r} \rho_{\beta} \xi_{\beta}=0$, then it results from (32) that:

$$
0=g\left(\xi_{\alpha}, \sum_{\beta=1}^{r} \rho_{\beta} \xi_{\beta}\right)=\rho_{\alpha}\left(1+\lambda_{\alpha}-\lambda_{\alpha}^{2}\right)
$$

for any $\alpha \in\{1, \ldots, r\}$. At the same time, because of the fact that $\lambda_{\alpha} \in(1-\phi, \phi)$, it is obvious that:

$$
1+\lambda_{\alpha}-\lambda_{\alpha}^{2} \neq 0
$$

Thus, it follows from (33) that $\rho_{\alpha}=0$ for any $\alpha \in\{1, \ldots, r\}$; that is, we get (a). Additionally, by a simple computation, we conclude from (19) that:

$$
\Phi\left(\xi_{\alpha}\right)=\left(1-\lambda_{\alpha}\right) \xi_{\alpha},
$$

from which (b) is correct.

Applying Lemma 3.9 to Theorem 3.4, Theorem 3.7, and Theorem 3.8, respectively, we have the following three theorems:

Theorem 3.10 Let $M$ be an n-dimensional submanifold of codimension $r$, isometrically immersed in any locally decomposable golden Riemannian manifold $(\bar{M}, \bar{g}, \bar{\Phi})$. If $a_{\alpha \beta}=\lambda_{\alpha} \delta_{\alpha \beta}, \lambda_{\alpha} \in(1-\phi, \phi)$ for any $\alpha, \beta \in\{1, \ldots, r\}$ and $\nabla \Phi=0$, then $M$ is totally geodesic.

Theorem 3.11 Let $M$ be an n-dimensional submanifold of codimension $r$, isometrically immersed in any locally decomposable golden Riemannian manifold $(\bar{M}, \bar{g}, \bar{\Phi})$. If $a_{\alpha \beta}=\lambda_{\alpha} \delta_{\alpha \beta}, \lambda_{\alpha} \in(1-\phi, \phi)$ for any $\alpha, \beta \in\{1, \ldots, r\}, \operatorname{tr}(\Phi)$ is constant, and $M$ is totally umbilical, then $M$ is totally geodesic. 
Theorem 3.12 Let $M$ be an n-dimensional submanifold of codimension $r$, isometrically immersed in any locally decomposable golden Riemannian manifold $(\bar{M}, \bar{g}, \bar{\Phi})$. If $a_{\alpha \beta}=\lambda_{\alpha} \delta_{\alpha \beta}, \lambda_{\alpha} \in(1-\phi, \phi)$ for any $\alpha, \beta \in\{1, \ldots, r\}, \operatorname{tr}(\Phi)$ is constant, and $\sum_{i=1}^{n}\left(\nabla_{e_{i}} \Phi\right) e_{i}=0$, then $M$ is minimal, where $\left\{e_{1}, \ldots, e_{n}\right\}$ is an orthonormal basis of the tangent space $T_{p} M$ at a point $p \in M$.

Lemma 3.13 Let $M$ be an $n$-dimensional submanifold of codimension $r$, isometrically immersed in any golden Riemannian manifold $(\bar{M}, \bar{g}, \bar{\Phi})$. If $\alpha_{\alpha \beta}=\delta_{\alpha \beta}$ for any $\alpha, \beta \in\{1, \ldots, r\}$, then the following assertions are satisfied:

(a) The tangent vector fields $\xi_{1}, \ldots, \xi_{r}$ are linearly independent,

(b) $\operatorname{tr}(\Phi)$ is constant.

Proof We suppose that $a_{\alpha \beta}=\delta_{\alpha \beta}$ for any $\alpha, \beta \in\{1, \ldots, r\}$. Then, by a direct calculation, it follows from (18) that:

$$
u_{\beta}\left(\xi_{\alpha}\right)=\varepsilon \delta_{\alpha \beta}
$$

On the other hand, we see from (20) that:

$$
g\left(\xi_{\alpha}, \xi_{\beta}\right)=\varepsilon u_{\beta}\left(\xi_{\alpha}\right)
$$

Thus, it is clear from (34) and (35) that:

$$
g\left(\xi_{\alpha}, \xi_{\beta}\right)=\delta_{\alpha \beta},
$$

which means that we have (a). Also, taking into account that $\operatorname{tr}\left(a_{\alpha \beta}\right)=\operatorname{tr}\left(\delta_{\alpha \beta}\right)=r$, we conclude from (30) that (b) holds.

Lemma 3.14 Let $M$ be an $n$-dimensional submanifold of codimension $r$, isometrically immersed in any golden Riemannian manifold $(\bar{M}, \bar{g}, \bar{\Phi})$. Then, the following expressions are equivalent:

(a) For any $\alpha, \beta \in\{1, \ldots, r\}, a_{\alpha \beta}=\delta_{\alpha \beta}$,

(b) For any $\alpha \in\{1, \ldots, r\}, \bar{\Phi}^{-1}\left(N_{\alpha}\right) \in \Gamma(T M)$.

Proof If $a_{\alpha \beta}=\delta_{\alpha \beta}$ for any $\alpha, \beta \in\{1, \ldots, r\}$, then we derive by a direct calculation from (6) that:

$$
\bar{\Phi}^{-1}\left(N_{\alpha}\right)=\varepsilon i_{*}\left(\xi_{\alpha}\right)
$$

which implies that $\bar{\Phi}^{-1}\left(N_{\alpha}\right) \in \Gamma(T M)$ for any $\alpha \in\{1, \ldots, r\}$. That is, we get (a) $\Rightarrow$ (b). Conversely, we suppose that $\bar{\Phi}^{-1}\left(N_{\alpha}\right) \in \Gamma(T M)$ for any $\alpha \in\{1, \ldots, r\}$. By means of (6), we have:

$$
\sum_{\beta=1}^{r}\left(a_{\alpha \beta}-\delta_{\alpha \beta}\right) N_{\beta}=0 .
$$

Then, as $\left\{N_{1}, \ldots, N_{r}\right\}$ is a local orthonormal frame of the normal bundle $T M^{\perp}$, we obtain:

$$
a_{\alpha \beta}=\delta_{\alpha \beta},
$$

which shows $(b) \Rightarrow(a)$. Consequently, the proof has been completed.

Using both of Lemmas 3.13 and 3.14 in Theorem 3.4, Theorem 3.7, and Theorem 3.8, respectively, we obtain the following three theorems:

Theorem 3.15 Let $M$ be an n-dimensional submanifold of codimension $r$, isometrically immersed in any locally decomposable golden Riemannian manifold $(\bar{M}, \bar{g}, \bar{\Phi})$. If $\bar{\Phi}^{-1}\left(N_{\alpha}\right) \in \Gamma(T M)$ for any $\alpha \in\{1, \ldots, r\}$ and $\nabla \Phi=0$, then $M$ is totally geodesic.

Theorem 3.16 Let $M$ be an n-dimensional submanifold of codimension $r$, isometrically immersed in any locally decomposable golden Riemannian manifold $(\bar{M}, \bar{g}, \bar{\Phi})$. If $\bar{\Phi}^{-1}\left(N_{\alpha}\right) \in \Gamma(T M)$ for any $\alpha \in\{1, \ldots, r\}$ and $M$ is totally umbilical, then $M$ is totally geodesic. 
Theorem 3.17 Let $M$ be an n-dimensional submanifold of codimension $r$, isometrically immersed in any locally decomposable golden Riemannian manifold $(\bar{M}, \bar{g}, \bar{\Phi})$. If $\bar{\Phi}^{-1}\left(N_{\alpha}\right) \in \Gamma(T M)$ for any $\alpha \in\{1, \ldots, r\}$ and $\sum_{i=1}^{n}\left(\nabla_{e_{i}} \Phi\right) e_{i}=0$, then $M$ is minimal, where $\left\{e_{1}, \ldots, e_{n}\right\}$ is an orthonormal basis of the tangent space $T_{p} M$ at a point $p \in M$.

Let $\left\{N_{1}, \ldots, N_{r}\right\}$ and $\left\{N_{1}^{\prime}, \ldots, N_{r}^{\prime}\right\}$ be two local orthonormal frames of the normal bundle $T M^{\perp}$. Then, the decomposition of the normal vector field $N_{\alpha}^{\prime}$ in the local orthonormal frame $\left\{N_{1}, \ldots, N_{r}\right\}$ is given by:

$$
N_{\alpha}^{\prime}=\sum_{\gamma=1}^{r} k_{\alpha}^{\gamma} N_{\gamma}
$$

for any $\alpha \in\{1, \ldots, r\}$, where $\left(k_{\alpha}^{\gamma}\right)$ is an orthogonal matrix of type $r \times r$ [1]. We write:

$$
\begin{aligned}
& u_{\alpha}^{\prime}=\sum_{\gamma=1}^{r} k_{\alpha}^{\gamma} u_{\gamma}, \\
& \xi_{\alpha}^{\prime}=\sum_{\gamma=1}^{r} k_{\alpha}^{\gamma} \xi_{\gamma}
\end{aligned}
$$

and

$$
a_{\alpha \beta}^{\prime}=\sum_{\gamma=1}^{r} \sum_{\delta=1}^{r} k_{\alpha}^{\gamma} a_{\gamma \delta} k_{\beta}^{\delta} .
$$

Then, using (36), (5) and (6) take the following forms:

$$
\bar{\Phi}\left(i_{*} X\right)=i_{*} \Phi(X)+\sum_{\alpha=1}^{r} u_{\alpha}^{\prime}(X) N_{\alpha}^{\prime}
$$

and

$$
\bar{\Phi}\left(N_{\alpha}^{\prime}\right)=\varepsilon i_{*}\left(\xi_{\alpha}^{\prime}\right)+\sum_{\beta=1}^{r} a_{\alpha \beta}^{\prime} N_{\beta}^{\prime}, \varepsilon= \pm 1,
$$

respectively.

Besides, (38) implies that if the tangent vector fields $\xi_{1}, \ldots, \xi_{r}$ are linearly independent (resp., linearly dependent), then the tangent vector fields $\xi_{1}^{\prime}, \ldots, \xi_{r}^{\prime}$ are also linearly independent (resp., linearly dependent). As the matrix element $a_{\alpha \beta}$ is symmetric in the indices $\alpha$ and $\beta$, it can be reduced to the form $a_{\alpha \beta}^{\prime}=\lambda_{\alpha} \delta_{\alpha \beta}$, where $\lambda_{\alpha}$ 's are the eigenvalues of the matrix $\left(a_{\alpha \beta}\right)_{r \times r}$ for any $\alpha, \beta \in\{1, \ldots, r\}$ [14].

Now, we suppose that the tangent vector fields $\stackrel{r}{\xi}_{1}, \ldots, \xi_{r}$ are linearly dependent. Taking account of (17) and (18), it seems that:

$$
u_{\beta}^{\prime}\left(\xi_{\alpha}^{\prime}\right)=\varepsilon \delta_{\alpha \beta}\left(1+\lambda_{\alpha}-\lambda_{\alpha}^{2}\right)
$$

for any $\alpha, \beta \in\{1, \ldots, r\}$. Hence, we obtain:

$$
u_{\alpha}^{\prime}\left(\xi_{\alpha}^{\prime}\right)=\varepsilon\left(1+\lambda_{\alpha}-\lambda_{\alpha}^{2}\right)
$$

and

$$
u_{\beta}^{\prime}\left(\xi_{\alpha}^{\prime}\right)=0, \alpha \neq \beta
$$

Furthermore, considering (19) in terms of the tangent vector fields $\xi_{\alpha}^{\prime}$ 's, we can easily find:

$$
\Phi\left(\xi_{\alpha}^{\prime}\right)=\left(1-\lambda_{\alpha}\right) \xi_{\alpha}^{\prime},
$$


which implies that $\xi_{\alpha}^{\prime}$ 's are eigenvectors of the induced structure $\Phi$ corresponding to eigenvalues $\left(1-\lambda_{\alpha}\right)$ 's for any $\alpha \in\{1, \ldots, r\}$.

For an index $h \in\{1, \ldots, r\}$, such that $\lambda_{h}^{2}=\lambda_{h}+1$, using (20) and (43), we obtain:

$$
g\left(\xi_{h}^{\prime}, \xi_{h}^{\prime}\right)=0
$$

from which we have $\xi_{h}^{\prime}=0$. Thus, it follows immediately from (41) that:

$$
\bar{\Phi}\left(N_{h}^{\prime}\right)=\lambda_{h} N_{h}^{\prime} .
$$

That is, $N_{h}^{\prime}$ is an eigenvector of the golden structure $\bar{\Phi}$ corresponding to the eigenvalue $\lambda_{h}$. If $\lambda_{k}^{2} \neq \lambda_{k}+1$, $k=1, \ldots, s \leq r$, then it is clear from (42) that the tangent vector fields $\xi_{1}^{\prime}, \ldots, \xi_{s}^{\prime}$ are linearly independent. Hence, we conclude from (40) and (41) that:

$$
\bar{\Phi}\left(i_{*} X\right)=i_{*}(\Phi(X))+\sum_{k=1}^{s} u_{k}^{\prime}(X) N_{k}^{\prime}, s \leq \min (r, n)
$$

and

$$
\left\{\begin{array}{l}
\bar{\Phi}\left(N_{k}^{\prime}\right)=\varepsilon i_{*}\left(\xi_{k}^{\prime}\right)+\lambda_{k} N_{k}^{\prime}, k=1, \ldots, s, s<r, s \leq n \\
\bar{\Phi}\left(N_{l}^{\prime}\right)=\lambda_{l} N_{l}^{\prime}, l=s+1, \ldots, r
\end{array}\right.
$$

where $\lambda_{\alpha}$ 's are eigenvalues of the matrix $\left(a_{\alpha \beta}\right)_{r \times r}$ for any $\alpha, \beta \in\{1, \ldots, r\}$, such that $\lambda_{k}^{2} \neq \lambda_{k}+1, k=1, \ldots, s$ and $\lambda_{l}^{2}=\lambda_{l}+1, l=s+1, \ldots, r$. In particular, if $s=r$, then we have:

$$
\bar{\Phi}\left(N_{\alpha}^{\prime}\right)=\varepsilon i_{*}\left(\xi_{\alpha}^{\prime}\right)+\lambda_{\alpha} N_{\alpha}^{\prime}, \lambda_{\alpha}^{2} \neq \lambda_{\alpha}+1, \alpha=1, \ldots, r .
$$

Lemma 3.18 Let $M$ be an n-dimensional submanifold of codimension $r$, isometrically immersed in any golden Riemannian manifold $(\bar{M}, \bar{g}, \bar{\Phi})$. Then, the tangent vector fields $\xi_{1}, \ldots, \xi_{r}$ are linearly independent if and only if the normal vector fields $N_{1}, \ldots, N_{r}$ are not eigenvectors of the golden structure $\bar{\Phi}$.

Proof Let $N$ be a unit normal vector field being an eigenvector of the golden structure $\bar{\Phi}$. We remark that the eigenvalues of the golden structure $\bar{\Phi}$ are the values $\phi$ and $1-\phi$ satisfying the algebraic equation $\lambda^{2}=\lambda+1$. We transform the local orthonormal frame $\left\{N_{1}, \ldots, N_{r}\right\}$ of the normal bundle $T M^{\perp}$ into another orthonormal frame $\left\{N_{1}^{\prime}, \ldots, N_{r}^{\prime}\right\}$. If $N_{r}^{\prime}=N$, then we obtain from (47) that:

$$
\bar{\Phi}(N)=\varepsilon i_{*}\left(\xi_{r}^{\prime}\right)+\lambda N .
$$

On the other hand, as $N$ is the eigenvector of the golden structure $\bar{\Phi}$, we have $\bar{\Phi}(N)=\lambda N$. It follows from (48) that $\xi_{r}^{\prime}=0$. As a result, the tangent vector fields $\xi_{1}, \ldots, \xi_{r}$ are linearly dependent.

Conversely, we suppose that the tangent vector fields $\xi_{1}, \ldots, \xi_{r}$ are not linearly independent. Then, it is possible to get a zero tangent vector field $\xi_{r}^{\prime}=0$ under a suitable transformation of the local orthonormal frame $\left\{N_{1}, \ldots, N_{r}\right\}$. Hence, for the normal vector field $N_{r}^{\prime}$ corresponding to the tangent vector field $\xi_{r}^{\prime}$, we see from (47) that $\bar{\Phi}\left(N_{r}^{\prime}\right)=\lambda N_{r}^{\prime}$. In the other words, $N_{r}^{\prime}$ is an eigenvector of the golden structure $\bar{\Phi}$. Consequently, the proof has been completed by method of reductio ad absurdum.

By use of Lemma 3.18 in Theorem 3.4, Theorems 3.7, and Theorem 3.8, respectively, we get the following three theorems:

Theorem 3.19 Let $M$ be an n-dimensional submanifold of codimension $r$, isometrically immersed in any locally decomposable golden Riemannian manifold $(\bar{M}, \bar{g}, \bar{\Phi})$. If the normal vector fields $N_{1}, \ldots, N_{r}$ are not eigenvectors of the golden structure $\bar{\Phi}$ and $\nabla \Phi=0$, then $M$ is totally geodesic.

Theorem 3.20 Let $M$ be an n-dimensional submanifold of codimension $r$, isometrically immersed in any locally decomposable golden Riemannian manifold $(\bar{M}, \bar{g}, \bar{\Phi})$. If the normal vector fields $N_{1}, \ldots, N_{r}$ are not eigenvectors of the golden structure $\bar{\Phi}, \operatorname{tr}(\Phi)$ is constant, and $M$ is totally umbilical, then $M$ is totally geodesic. 
Theorem 3.21 Let $M$ be an n-dimensional submanifold of codimension $r$, isometrically immersed in any locally decomposable golden Riemannian manifold $(\bar{M}, \bar{g}, \bar{\Phi})$. If the normal vector fields $N_{1}, \ldots, N_{r}$ are not eigenvectors of the golden structure $\bar{\Phi}, \operatorname{tr}(\Phi)$ is constant, and $\sum_{i=1}^{n}\left(\nabla_{e_{i}} \Phi\right) e_{i}=0$, then $M$ is minimal, where $\left\{e_{1}, \ldots, e_{n}\right\}$ is an orthonormal basis of the tangent space $T_{p} M$ at a point $p \in M$.

Lemma 3.22 Let $M$ be an $n$-dimensional submanifold of codimension $r$, isometrically immersed in any golden Riemannian manifold $(\bar{M}, \bar{g}, \bar{\Phi})$. We assume that $\lambda_{k}^{2} \neq \lambda_{k}+1, k=1, \ldots$, s and $\lambda_{A}^{2}=\lambda_{A}+1, A=s+1, \ldots, n$, where $s<r$ and $s \leq n$. If $a_{\alpha \beta}=\lambda_{\alpha} \delta_{\alpha \beta}$ for any $\alpha, \beta \in\{1, \ldots, r\}$, then the trace of the induced structure $\Phi$ is given by:

$$
\operatorname{tr}(\Phi)=s-\sum_{k=1}^{s} \lambda_{k}+\sum_{A=s+1}^{n} \lambda_{A}
$$

where $\lambda_{A} \in\{\phi, 1-\phi\}$.

Proof We define two matrices $\bar{U}$ and $L$ by:

$$
\bar{U}=\left(\xi_{1}^{\prime} \cdots \xi_{s}^{\prime} \eta_{s+1}^{\prime} \cdots \eta_{n}^{\prime}\right)
$$

and

$$
L=\left(\begin{array}{cc}
\left(1-\lambda_{k}\right) \delta_{k l} & 0 \\
0 & \lambda_{A} \delta_{A B}
\end{array}\right)
$$

respectively. Then, it seems clearly from (44) that:

$$
(\Phi) \bar{U}=\bar{U} L,
$$

where $(\Phi)$ is the matrix corresponding to the induced structure $\Phi$. Hence, using the fact that $\operatorname{det}(\bar{U}) \neq 0$, we obtain:

$$
(\Phi)=\bar{U} L \bar{U}^{-1}
$$

from which

$$
\operatorname{tr}(\Phi)=\operatorname{tr}\left(\bar{U} L \bar{U}^{-1}\right)=\operatorname{tr}(L)=s-\sum_{k=1}^{s} \lambda_{k}+\sum_{A=s+1}^{n} \lambda_{A}
$$

Theorem 3.23 Let $M$ be an $n$-dimensional submanifold of codimension $r$, isometrically immersed in any locally decomposable golden Riemannian manifold $(\bar{M}, \bar{g}, \bar{\Phi})$. We suppose that $\lambda_{k}^{2} \neq \lambda_{k}+1, k=1, \ldots, s$ and $\lambda_{l}^{2}=\lambda_{l}+1, l=s+1, \ldots, r$, where $s<r$ and $s \leq n$. If $a_{\alpha \beta}=\lambda_{\alpha} \delta_{\alpha \beta}$ for any $\alpha, \beta \in\{1, \ldots, r\}$ and $\nabla \Phi=0$, then we have $h_{k}=0, k=1, \ldots, s$.

Proof Since the induced structure $\Phi$ is parallel with respect to the induced connection $\nabla$, we derive from (11), (20), and (23) that:

$$
\sum_{k=1}^{s} u_{k}^{\prime}(Y) h_{k}(X, Z)=-\sum_{k=1}^{s} u_{k}^{\prime}(Z) h_{k}(X, Y)
$$

for any vector fields $X, Y, Z \in \Gamma(T M)$. Also, taking into account (10), (50) is given by:

$$
\sum_{k=1}^{s} u_{k}^{\prime}(Y) h_{k}(X, Z)=\sum_{k=1}^{s} u_{k}^{\prime}(Z) h_{k}(X, Y)
$$


for any vector fields $X, Y, Z \in \Gamma(T M)$. Thus, it results from (50) and (51) that:

$$
\sum_{k=1}^{s} u_{k}^{\prime}(Y) h_{k}(X, Z)=0 .
$$

On the other hand, we recall that if $\lambda_{k}^{2} \neq \lambda_{k}+1, k=1, \ldots, s$, the tangent vector fields $\xi_{1}^{\prime}, \ldots, \xi_{s}^{\prime}$ are linearly independent, and so are the differential 1-forms $u_{k}^{\prime}$ 's. Hence, it follows from (52) that $h_{k}=0, k=1, \ldots, s$.

Lemma 3.24 Let $M$ be an n-dimensional submanifold of codimension $r$, isometrically immersed in any locally decomposable golden Riemannian manifold $(\bar{M}, \bar{g}, \bar{\Phi})$. We assume that $\lambda_{k}^{2} \neq \lambda_{k}+1, k=1, \ldots, s$ and $\lambda_{l}^{2}=\lambda_{l}+1, l=s+1, \ldots, r$, where $s<r$ and $s \leq n$. If $a_{\alpha \beta}=\lambda_{\alpha} \delta_{\alpha \beta}$ for any $\alpha, \beta \in\{1, \ldots, r\}$ and $\operatorname{tr}(\Phi)$ is constant, then for any vector field $X \in \Gamma(T M)$, we have:

$$
\sum_{k=1}^{s} h_{k}\left(X, \xi_{k}^{\prime}\right)=0
$$

and

$$
\sum_{k=1}^{s} A_{k} \xi_{k}^{\prime}=0
$$

Proof In (26), by taking $\alpha=\beta$, we get:

$$
2 \varepsilon h_{k}\left(X, \xi_{k}^{\prime}\right)+\nabla_{X} \lambda_{\alpha}+2 \sum_{\gamma=1}^{r} a_{\alpha \gamma} l_{\gamma \alpha}(X)=0
$$

for any vector field $X \in \Gamma(T M)$. Then, summing over $\alpha$ in (55), we have:

$$
2 \varepsilon \sum_{k=1}^{s} h_{k}\left(X, \xi_{k}^{\prime}\right)+\nabla_{X} \sum_{\alpha=1}^{r} \lambda_{\alpha}+2 \sum_{\alpha=1}^{r} \sum_{\gamma=1}^{r} a_{\alpha \gamma} l_{\gamma \alpha}(X)=0 .
$$

By means of (13) and (17), (56) is reduced to the form:

$$
2 \varepsilon \sum_{k=1}^{s} h_{k}\left(X, \xi_{k}^{\prime}\right)+\nabla_{X} \sum_{\alpha=1}^{r} \lambda_{\alpha}=0 .
$$

On the other hand, since $\operatorname{tr}(\Phi)$ is constant, it is clear from (49) that so is $\sum_{\alpha=1}^{r} \lambda_{\alpha}$. Hence, (53) follows from (57). Also, (11) states that (53) is equivalent to (54). As a result, the proof has been completed.

Theorem 3.25 Let $M$ be an n-dimensional submanifold of codimension $r$, isometrically immersed in any locally decomposable golden Riemannian manifold $(\bar{M}, \bar{g}, \bar{\Phi})$. We suppose that $\lambda_{k}^{2} \neq \lambda_{k}+1, k=1, \ldots, s$ and $\lambda_{l}^{2}=\lambda_{l}+1, l=s+1, \ldots, r$, where $s<r$ and $s \leq n$. If $a_{\alpha \beta}=\lambda_{\alpha} \delta_{\alpha \beta}$ for any $\alpha, \beta \in\{1, \ldots, r\}, \operatorname{tr}(\Phi)$ is constant, and $M$ is totally umbilical, then $h_{k}=0, k=1, \ldots, s$.

Proof As $M$ is totally umbilical, there are constants $\sigma_{k}$ 's, such that:

$$
h_{k}=\sigma_{k} g
$$

for each $k=1, \ldots, s$. On the other hand, since all the conditions of Lemma 3.24 are satisfied, (53) is valid. Then, using (58) in (53), we get:

$$
0=\sum_{k=1}^{s} h_{\alpha}\left(X, \xi_{k}^{\prime}\right)=g\left(X, \sum_{k=1}^{s} \sigma_{k} \xi_{k}^{\prime}\right)
$$


from which we have:

$$
\sum_{k=1}^{s} \sigma_{k} \xi_{k}^{\prime}=0
$$

On account of the fact that $\lambda_{k}^{2} \neq \lambda_{k}+1, k=1, \ldots, s$, the tangent vector fields $\xi_{1}^{\prime}, \ldots, \xi_{s}^{\prime}$ are linearly independent. Thus, it results from (58) that $h_{k}=0, k=1, \ldots, s$.

Theorem 3.26 Let $M$ be an n-dimensional submanifold of codimension $r$, isometrically immersed in any locally decomposable golden Riemannian manifold $(\bar{M}, \bar{g}, \bar{\Phi})$. We assume that $\lambda_{k}^{2} \neq \lambda_{k}+1, k=1, \ldots, s$ and $\lambda_{l}^{2}=\lambda_{l}+1, l=s+1, \ldots, r$, where $s<r$ and $s \leq n$. If $a_{\alpha \beta}=\lambda_{\alpha} \delta_{\alpha \beta}$ for any $\alpha, \beta \in\{1, \ldots, r\}, \operatorname{tr}(\Phi)$ is constant, and $\sum_{i=1}^{n}\left(\nabla_{e_{i}} \Phi\right) e_{i}=0$, then $\sum_{i=1}^{n} h_{k}\left(e_{i}, e_{i}\right)=0, k=1, \ldots, s$, where $\left\{e_{1}, \ldots, e_{n}\right\}$ is an orthonormal basis of the tangent space $T_{p} M$ at a point $p \in M$.

Proof For any $i \in\{1, \ldots, n\}$, choosing $X_{p}=Y_{p}=e_{i}$ at the point $p \in M$ in (23), then we have:

$$
\left(\nabla_{e_{i}} \Phi\right) e_{i}=\varepsilon \sum_{k=1}^{s} h_{k}\left(e_{i}, e_{i}\right) \xi_{k}^{\prime}+\sum_{k=1}^{s} u_{k}^{\prime}\left(e_{i}\right) A_{k} e_{i}
$$

Thus, summing over $i$, from the fact that $\sum_{i=1}^{n} u_{k}^{\prime}\left(e_{i}\right) e_{i}=\varepsilon \xi_{k}^{\prime}$ and the assumption that $\sum_{i=1}^{n}\left(\nabla_{e_{i}} \Phi\right) e_{i}=0$, we get:

$$
0=\sum_{i=1}^{n}\left(\nabla_{e_{i}} \Phi\right) e_{i}=\varepsilon \sum_{k=1}^{s}\left(\sum_{i=1}^{n} h_{k}\left(e_{i}, e_{i}\right) \xi_{k}^{\prime}+A_{k} \xi_{k}^{\prime}\right)
$$

On the other hand, as all the conditions of Lemma 3.24 hold in the hypothesis, (54) is verified. Hence, from (54) and (60), we obtain:

$$
\sum_{k=1}^{s}\left(\sum_{i=1}^{n} h_{k}\left(e_{i}, e_{i}\right)\right) \xi_{k}^{\prime}=0
$$

which shows that $\sum_{i=1}^{n} h_{k}\left(e_{i}, e_{i}\right)=0$ by virtue of the fact that the tangent vector fields $\xi_{1}^{\prime}, \ldots, \xi_{s}^{\prime}$ are linearly independent.

Open Access This article is licensed under a Creative Commons Attribution 4.0 International License, which permits use, sharing, adaptation, distribution and reproduction in any medium or format, as long as you give appropriate credit to the original author(s) and the source, provide a link to the Creative Commons licence, and indicate if changes were made. The images or other third party material in this article are included in the article's Creative Commons licence, unless indicated otherwise in a credit line to the material. If material is not included in the article's Creative Commons licence and your intended use is not permitted by statutory regulation or exceeds the permitted use, you will need to obtain permission directly from the copyright holder. To view a copy of this licence, visit http://creativecommons.org/licenses/by/4.0/.

\section{References}

1. Adati, T.: Submanifolds of an almost product Riemannian manifold. Kodai Math. J. 4(2), 327-343 (1981)

2. Ahmad, M.; Qayyoom, M.A.: On submanifolds in a Riemannian manifold with golden structure. Turk. J. Math. Comput. Sci. 11(1), 8-23 (2019)

3. Blaga, A.M.: The geometry of golden conjugate connections. Sarajevo J. Math. 10(23), 237-245 (2014)

4. Blaga, A.M.; Hrețcanu, C.E.: Invariant, anti-invariant and slant submanifolds of a metallic Riemannian manifold. Novi Sad J. Math. 48(2), 55-80 (2018)

5. Crâşmăreanu, M.C.; Hreţcanu, C.E.: Golden differential geometry. Chaos Solitons Fractals 38(5), 1229-1238 (2008)

6. Erdoğan, F.E.; Yıldırım, C.: On a study of the totally umbilical semi-invariant submanifolds of golden Riemannian manifolds. J. Polytechnic 21(4), 967-970 (2018) 
7. Gezer, A.; Cengiz, N.; Salimov, A.: On integrability of golden Riemannian structures. Turk. J. Math. 37(4), 693-703 (2013)

8. Gherici, B.: Induced structures on golden Riemannian manifolds. Beitr. Algebra. Geom. 59(4), 761-777 (2018)

9. Gök, M., Keleş, S., Kılıç, E.: Some characterizations of semi-invariant submanifolds of golden Riemannian manifolds. Mathematics 7(12), Article ID 1209, 12 pages (2019)

10. Gök, M.; Keleş, S.; Kılıç, E.: Invariant submanifolds in golden Riemannian manifolds. Commun. Fac. Sci. Univ. Ank. Ser. A1 Math. Stat. 69(2), 125-138, (2020)

11. Gök, M.; Keleş, S.; Kılıç, E.: Schouten and Vrănceanu connections on golden manifolds. Int. Electron. J. Geom. 12(2), 169-181 (2019)

12. Hreţcanu, C.E., Blaga, A.M.: Slant and semi-slant submanifolds in metallic Riemannian manifolds. J. Funct. Spaces 2018, Article ID 2864263, 13 pages (2018)

13. Hreţcanu, C.E.; Cr âşmăreanu, M.C.: On some invariant submanifolds in a Riemannian manifold with golden structure. An. Ştiinţ. Univ. Al. I. Cuza Iaş. Mat. (N.S.) 53(suppl. 1), 199-211 (2007)

14. Hreţcanu, C.E.; Blaga, A.M.: Hemi-slant submanifolds in metallic Riemannian manifolds. Carpathian J. Math. 35(1), 59-68 (2019)

15. Hreţcanu, C.E.; Crâşmăreanu, M.C.: Metallic structures on Riemannian manifolds. Rev. Un. Mat. Argentina 54(2), 15-27 (2013)

16. Hreţcanu, C.E.; Crâşmăreanu, M.C.: Applications of the golden ratio on Riemannian manifolds. Turk. J. Math 33(2), 179-191 (2009)

17. Kalia, S.: The generalizations of the golden ratio: their powers, continued fractions, and convergents. MIT Mathematics. http://math.mit.edu/research/highschool/primes/papers.php (2011). Accessed 23 Dec 2011

18. Özkan, M.: Prolongations of golden structures to tangent bundles. Differ. Geom. Dyn. Syst. 16, 227-238 (2014)

19. Özkan, M.; Peltek, B.: A new structure on manifolds: silver structure. Int. Electron. J. Geom. 9(2), 59-69 (2016)

20. Pandey, P.K.: Sameer: Bronze differential geometry. J. Sci. Arts 4(45), 973-980 (2018)

21. Perktaş, S.Y.: Submanifolds of almost poly-Norden Riemannian manifolds. Turk. J. Math. 44(1), 31-49 (2020)

22. Şahin, B.: Almost poly-Norden manifolds. Int. J. Maps Math. 1(1), 68-79 (2018)

23. Şahin, B.; Akyol, M.A.: Golden maps between golden Riemannian manifolds and constancy of certain maps. Math. Commun. 19(2), 333-342 (2014)

24. Yano, K.; Kon, M.: Structures on Manifolds. World Scientific, Singapore (1984)

Publisher's Note Springer Nature remains neutral with regard to jurisdictional claims in published maps and institutional affiliations. 